\title{
Mobile robot control on uneven and slippery ground: an adaptive approach based on a multi-model observer
}

\author{
Roland Lenain ${ }^{1}$ and Benoit Thuilot ${ }^{2,3}$
}

\begin{abstract}
This paper proposes an algorithm dedicated to off-road mobile robot path tracking at high speed. In order to ensure a high accuracy, a predictive and adaptive approach is developed to face the various perturbations due to this context (mainly the bad grip conditions and the terrain geometry). The control law is based on previous work, and requires the knowledge of sideslip angles, which cannot be directly measured. As a result, an observer based on two levels of modeling (kinematic and dynamic) is proposed to ensure a relevant and fast estimation. If the kinematic part is independent from the terrain geometry, the dynamic model used in this paper requires to take explicitly into account the influence of the terrain geometry on mobile robot dynamic. It is achieved by the introduction of the lateral robot inclination, which is on-line estimated via a Kalman filter and integrated into the dynamical model. The advantages of the proposed contribution to path tracking control are investigated through full-scale experiments achieved at high speed (up to $6 \mathrm{~m} / \mathrm{s}$ ) on an uneven and grass field.
\end{abstract}

\section{INTRODUCTION}

The growing social demand in terms of security and productivity makes new needs arise for off-road mobile robots. The increasing capabilities of unmanned vehicles indeed indicate the possible benefits in various fields of applications, such as transportation, defense or agriculture. Nevertheless, in order to be efficient, such automatic devices must be accurate and fast, whatever the ground conditions (nature and geometry) and the path they must follow. Despite numerous work in off-road mobile robotics (see for instance [1]), the accurate control of mobile robots in natural environment is still a challenging problem, especially at high speed. High dynamics and varying grip conditions encountered indeed constitute important perturbations, which have to be accounted in order to preserve a satisfactory accuracy.

Specifically, from a path tracking point of view, classical control laws (such as initiated in [2]), assuming rolling without sliding conditions, are not convenient. In such a context, they indeed lead to large tracking errors. As a result, new methods have to be considered in order to preserve the motion accuracy, and several strategies are investigated to face this problem. Robust control (see for instance [3] or [4]), considering sliding as a perturbation to be rejected, can be applied, improving tracking error, but appearing to be conservative with an oscillating behavior. If this can be acceptable

\footnotetext{
${ }^{1} \mathrm{R}$. Lenain is with Irstea, TSCF Unit, Centre of ClermontFerrand, 24 avenue des Landais BP50085, Aubiere Cedex, France roland.lenaindirstea.fr

${ }^{2}$ B. Thuilot is with Institut Pascal, Université Blaise Pascal, BP 10448, 63000 Clermont-Ferrand, France

${ }^{3} \mathrm{~B}$. Thuilot is also with CNRS - UMR 6602, 63177 Aubiere, France benoit.thuilotelasmea.univ-bpclermont. fr
}

when moving slowly, the use of such approaches at high speed may lead to instability. A second way consists in taking sliding explicitly into account via control laws based solely on dynamic models (see for instance [5] or [6]). Nevertheless it requires the knowledge of numerous parameters which appear to be varying in off-road conditions, as well as the use of huge perception systems [11].

In this paper, an alternative control strategy, based on adaptive and predictive control principles is proposed. More precisely, the bad grip conditions are accounted inside the control law by sideslip angles introduced in an extended kinematic model (such as initiated by [7] and generalized in [8]). These angles can be on-line estimated via an observer based only on the proposed kinematic representation [9]. This is relevant at relatively low speed (below $4 \mathrm{~m} / \mathrm{s}$ with the experimental testbed described in this paper) and particularly suitable, as it does not require a huge perception system. Nevertheless, such an approach appears to be not reactive enough when moving at high speed. As a consequence, a partial dynamic observer used together with the previous control strategy has been proposed in [10], using a 2D dynamic model. It permits to obtain accurate performances on a flat ground at high speed (up to $8 \mathrm{~m} / \mathrm{s}$ ), but does not provide satisfying results when the mobile robot moves on an uneven ground. The lateral contribution of the gravity during a motion on an uneven ground is indeed neglected in the proposed model and does not permit a correct estimation of sideslip angles. In order to ensure an accurate path tracking at high speed on sloping fields, such a multi-model observer strategy is here generalized. A new observer, allowing to take also into account the influence of terrain geometry on mobile robot behavior, is proposed in this paper. This is achieved by considering the robot roll angle, and therefore requires its online estimation.

The different levels of modeling used in this paper are first presented. The paper then details the observation algorithm in a second part. In particular, the different steps for the dynamic estimation of sideslip angles accounting for the roll angle are described: preliminary kinematic estimation, robot lateral inclination estimation, cornering stiffness adaptation, and finally the observer based on adapted dynamic model. After recalling briefly the control law in a third part, the capabilities of the proposed observer are investigated through full-scale experiments.

\section{RoBot MODELS}

In order to permit an accurate off-road path tracking at high speed, the proposed control algorithm takes advantage 
of several levels of representation. In this section, the extended kinematic point of view is first detailed. Secondly, the dynamical model accounting for the influence of the terrain geometry is described.

\section{A. Extended kinematic model}

The extended kinematic model, enabling to preserve a kinematic structure while describing the influence of sliding on the robot motion is depicted in figure 1. As achieved in [12], the robot is here considered as a bicycle. As the objective is to develop a path tracking algorithm, the motion is described with respect to a reference trajectory $\Gamma$. The robot position and orientation are then defined in terms of lateral and angular deviations : $y$ and $\tilde{\theta}$. Control variables are the velocity $v$ (here considered as a measured parameter - the velocity control is not investigated in this paper), and the front steering angle $\delta_{F}$. In addition to these classical

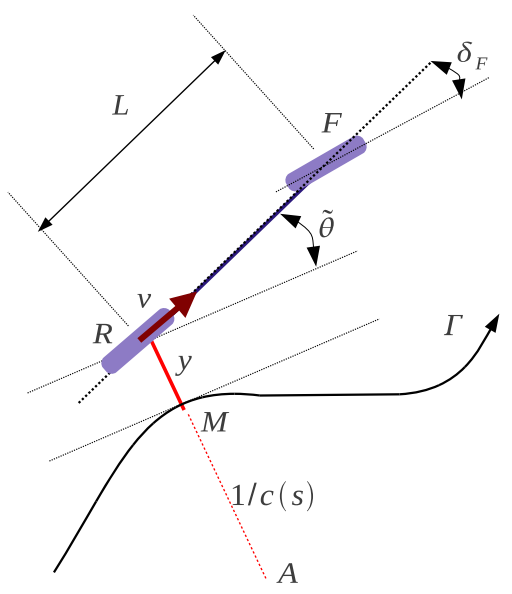

Fig. 1. Extended kinematic model

variables, two sideslip angles representative of wheel sliding are added: $\beta_{F}$ and $\beta_{R}$, for the front and the rear axle respectively. A sideslip angle is representative of the difference between the tire and the actual speed vector orientations. Using these notations, the expression of the mobile robot kinematic model, the computation of which is detailed in [9], can be expressed as:

$$
\left\{\begin{aligned}
\dot{s} & =v \frac{\cos \left(\tilde{\theta}+\beta_{R}\right)}{1-c(s) y} \\
\dot{y} & =v \sin \left(\tilde{\theta}+\beta_{R}\right) \\
\dot{\tilde{\theta}} & =v\left[\cos \left(\beta_{R}\right) \lambda_{1}-\lambda_{2}\right]
\end{aligned}\right.
$$

with : $\lambda_{1}=\frac{\tan \left(\delta_{F}-\beta_{F}\right)-\tan \left(\beta_{R}\right)}{L}, \lambda_{2}=\frac{c(s) \cos \left(\tilde{\theta}+\beta_{R}\right)}{1-c(s) y}$ This kinematic representation permits to derive easily a control law, as discussed in section IV. Moreover, the estimation of sideslip angles at low speed can be proceeded thanks to this model (see section III), but dynamical effects are then neglected. As a consequence, a dynamic model is required for sideslip angle estimation when moving fast.

\section{B. Dynamic model}

In order to account for mobile robot dynamics encountered at high speed, the dynamical model depicted in figure 2(a) is considered.

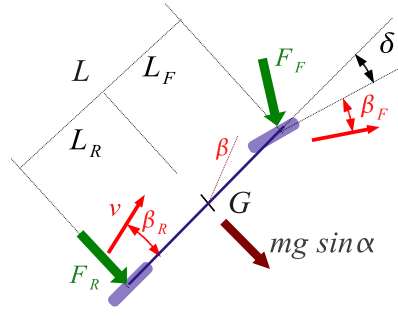

(a) Yaw frame

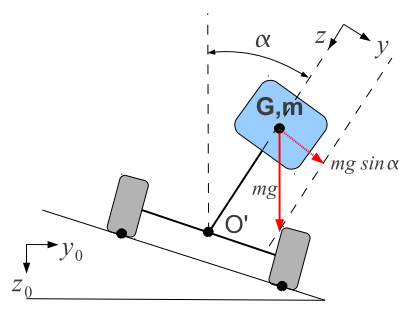

(b) Roll frame ( $\alpha$ definition)
Fig. 2. Dynamic model used for sideslip angle observation

This model is also based on the bicycle assumption, as achieved in [13]. In addition to variables used for the kinematic representation, the global sideslip angle $\beta$ and the robot global heading $\theta$ are introduced. Since the path tracking task is here supposed to be performed with a slow varying velocity, the longitudinal forces are neglected. As a result, only the lateral component of contact forces is considered: $F_{F}$ and $F_{R}$ (for the front and rear axles). An expression of these forces can be obtained by complex interaction models, such as Pacejka ([14]), which introduces an important number of parameters, pending on contact properties. These properties are moreover varying in off-road context, and such models then appear to be hardly tractable. In order to preserve the simplicity of the contact model, each of the contact forces is considered to be proportional to the corresponding sideslip angle, such as:

$$
\left\{\begin{array}{l}
F_{F}=C_{F}(.) \beta_{F} \\
F_{R}=C_{R}(.) \beta_{R}
\end{array}\right.
$$

However, in order to account for contact variability and tire non-linearity, coefficients $C_{F, R}($.$) (cornering stiffnesses),$ are considered as varying, and have then to be on-line estimated. In addition, the weight has also an influence on the robot dynamic. Indeed, when moving on a sloping field, the gravity has a non-null contribution on the robot yaw frame. Since a lateral motion control is solely investigated in this paper, only the contribution of the gravity to the lateral motion is here considered. This is achieved by introducing the term m.g.sin $\alpha$, where $\alpha$ is the roll angle of the suspended robot mass (as described in figure 2(b)). This angle $\alpha$ is then not representative of the terrain bank angle, but expresses the lateral robot inclination, and reflects the orientation of the gravity in the robot frame $(G, y, z)$.

Finally, the model depicted in figure 2(a) also requires the knowledge of dynamic parameters: the robot mass $m$, vertical inertia $I_{z}$ and the longitudinal position of the centre of gravity (point $G$ ) described by the front and rear half wheelbases $L_{F}$ and $L_{R}$. Using these notations and assumptions, the yaw dynamic model can be expressed as (see [15] for details): 


$$
\left\{\begin{aligned}
\ddot{\theta}= & \frac{1}{I_{z}}\left(-L_{F} F_{F} \cos \left(\delta_{F}\right)+L_{R} F_{R}\right) \\
\dot{\beta}= & -\frac{1}{v m}\left(F_{F} \cos \left(\beta-\delta_{F}\right)+F_{R} \cos (\beta)\right) \\
& +\frac{g \sin (\alpha)}{v}-\dot{\theta} \\
\beta_{R}= & \arctan \left(\tan \beta-\frac{L_{R} \dot{\theta}}{v \cos (\beta)}\right) \\
\beta_{F}= & \arctan \left(\tan \beta+\frac{L_{F} \dot{\theta}}{v \cos (\beta)}\right)-\delta_{F}
\end{aligned}\right.
$$

As it can be seen on equations (3), this model exists if the velocity is not null, which is supposed to be true in the path tracking application here considered. It can also be noticed that sideslip angles are shared variables between extended kinematic and dynamic models.

\section{MUlti-MOdEL OBSERVER}

As mentioned before, the extended kinematic model (1) permits to compute the control law expression for front steering wheels as soon as sideslip angles $\beta_{F}$ and $\beta_{R}$ are known. The other variables $v, \delta, y$ and $\tilde{\theta}$ can be measured by the sensors on-boarded, described in section V-A. As there is no available measures for sideslip angles, an indirect estimation must be achieved. It must be accurate and reactive enough to be efficient at high speed.

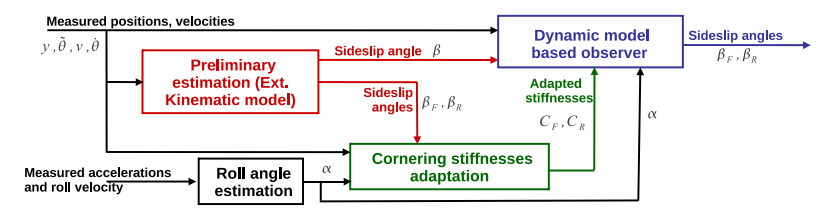

Fig. 3. Global scheme of observation strategy

This is achieved thanks to the observation strategy described by the scheme depicted in figure 3 . The global observer is decomposed into several parts. First, a preliminary observation based on the kinematic model (1) is achieved. An estimation of $\beta_{F}$ and $\beta_{R}$ is then obtained thanks to the convergence of kinematic model outputs to the measured lateral and angular deviations, as detailed in section III-A. As the effect of the robot inclination is reflected in these measurements, this observer naturally accounts for the terrain geometry. Alas, dynamical effects are neglected, leading to a slow-varying sideslip angle estimation, unsuitable when moving at high speed. Dynamic model (3) must be used to allow a faster adaptation. Nevertheless, such a model must be fed with relevant values of cornering stiffnesses $C_{F}$ and $C_{R}$, and has to take explicitly into account the influence of terrain geometry. This is achieved thanks to the contribution of gravity in the robot lateral behavior: it can indeed be noticed that if the term $m g \sin \alpha$ is neglected, model (3) cannot reflect the influence of slope on sideslip angles and these latter variables would be misestimated. As a result, the mobile robot roll angle is first estimated as detailed in section III-B. Together with the preliminary sideslip angle observer (supplying a reference value, relevant in steady state), the adaptation of the dynamic model parameters $\left(C_{F}\right.$ and $C_{R}$ ) can then be processed (as described in section IIIC). The dynamic model is then totally known, and is finally used to build an observer for the fast estimation of the required sideslip angles. This last step is depicted by the box "Dynamic model-based observer" in figure 3, and the equations are detailed in section III-D.

\section{A. Sideslip angle kinematic-based observer}

This preliminary observation using only model (1) is detailed in [9], where several experimental results can be found at relatively low speed (below $4 \mathrm{~m} / \mathrm{s}$ ) and for different kinds of terrain geometry. Detailed equations are then not presented here, but the general description is summarized in figure 4 . The objective is to compute the front and rear

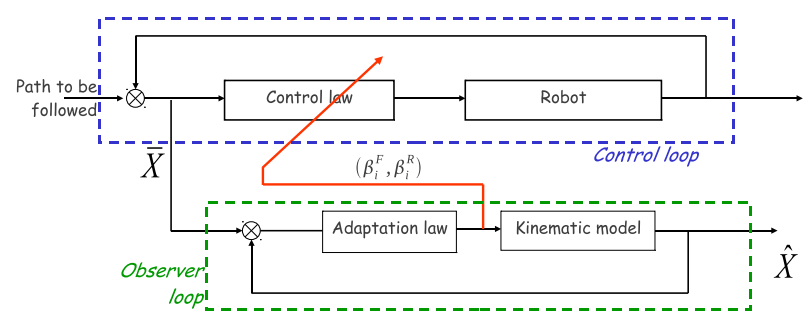

Fig. 4. Extended kinematic model-based observer

sideslip angles hereafter denoted $\bar{\beta}_{F}$ and $\bar{\beta}_{R}$ ensuring the convergence of the observed model outputs $\left(\hat{X}=\left[\begin{array}{cc}\hat{y} & \hat{\tilde{\theta}}\end{array}\right]\right)$ to the measured outputs $\bar{X}$. Sideslip angles thus estimated are then representative of any differences between kinematic description and the measured robot motion, mainly due to sliding. They take implicitly into account the different phenomena inducing sliding (motion on slope or bends on a low grip ground). However, such an observer does not account for dynamical behavior on sideslip angle evolution and is then low reactive, depreciating the tracking accuracy when moving fast. As a result, an observer based on a dynamic model is required to move at higher speed. Nevertheless, this preliminary observer supplies reference steady state values (and especially the global sideslip angles $\bar{\beta}$ ) allowing the adaptation of slow-varying parameters of a dynamic model, which can then be used to build a sideslip angle observer based on a dynamic model.

\section{B. Lateral inclination estimation}

Roll angle $\alpha$ could be directly measured using an inclinometer. However the estimation is here obtained by a Kalman filter, taking advantage of lateral and vertical accelerations (denoted respectively $a_{y}$ and $a_{z}$ ), together with roll rate $\dot{\alpha}$, all obtained thanks to a low cost IMU (see section V-A for a list of sensors). This sensor is located on the suspended mass in the frame depicted in figure 2(b), and is then able to catch the gravitational acceleration along both $y$ and $z$ axes. This permits a dynamic estimation of the roll angle, avoiding the delays due to inclinometer technology. Considering the definition of $\alpha$, a measurement of this angle may be obtained directly from the accelerations thanks to equation (4).

$$
\bar{\alpha}=\arctan \frac{a_{y}-v \dot{\theta}}{a_{z}}
$$


where the term $v \dot{\theta}$ constitutes the inertial contribution to the measured lateral acceleration $a_{y}$, which is not representative of the robot lateral inclination. Since equation (4) can lead to a noisy signal and considering the availability of a measurement of the roll angle derivative $\dot{\bar{\alpha}}$ thanks to the onboarded IMU (see section V-A), a Kalman filter is designed. Let us define by $k$ the current iteration. The estimated roll angle $\hat{\alpha}_{[k \mid k]}$ can be obtained as follows:

$$
\begin{aligned}
\hat{\alpha}_{[k \mid k-1]} & =\hat{\alpha}_{[k-1 \mid k-1]}+T \dot{\bar{\alpha}}_{[k]} \\
\hat{\alpha}_{[k \mid k]} & =\hat{\alpha}_{[k \mid k-1]}+K_{\alpha}\left(\bar{\alpha}_{[k]}-\hat{\alpha}_{[k \mid k-1]}\right)
\end{aligned}
$$

where $T$ is the sampling period of the IMU, and $K_{\alpha}$ is the Kalman gain obtained from the Kalman filtering theory, not here detailed. The filter is initialized using the first measurements of $\bar{\alpha}$ supplied by equation (4), as the robot does not move during one second before starting. Using the filter (5), an estimation of the roll angle $\hat{\alpha}_{[k \mid k]}$ is available, hereafter denoted $\alpha$.

\section{Cornering stiffness adaptation}

Once $\alpha$, considered as a measured parameter, and $\bar{\beta}$ are known, the only unknowns in model (3) are the cornering stiffnesses $C_{F}$ and $C_{R}$. If these parameters are assumed slow varying with respect to the mobile robot dynamic, the low reactive estimation of global sideslip angle $\bar{\beta}$ together with the measurement of the yaw rate $\dot{\theta}$ allow the proper adaptation of $C_{F}$ and $C_{R}$. These two variables are here regarded as the outputs of dynamic model (3), while $\alpha$ is required in order to preserve the model relevancy with respect to the robot motion when moving on slope. Cornering stiffness estimation is achieved in two steps. First, front and rear lateral forces are derived from a model whose state vector is $X_{1}=\left[\begin{array}{ll}\dot{\theta}_{1} & \beta_{1}\end{array}\right]^{T}$, i.e. composed of the yaw rate and the global sideslip angle. Using on-boarded sensors and the estimation $\bar{\beta}$ of the global sideslip angle, a measurement $\bar{X}_{1}$ of this state is available. In view of model (3), the state derivative can be written as :

$$
\dot{X}_{1}=A_{1}\left(X_{1}\right)+B_{1}\left(\delta_{F}\right)\left[\begin{array}{ll}
F_{F} & F_{R}
\end{array}\right]^{T}
$$

where:

$$
\begin{gathered}
A_{1}\left(X_{1}\right)=\left[\begin{array}{cc}
0 \\
\frac{g \sin \alpha}{v}-\dot{\theta}_{1}
\end{array}\right] \\
B_{1}\left(\delta_{F}\right)=\left[\begin{array}{cc}
-\frac{L_{F} \cos \delta_{F}}{I_{z}} & \frac{L_{R}}{I_{z}} \\
-\frac{\cos \delta_{F}}{v m} & -\frac{1}{v m}
\end{array}\right]
\end{gathered}
$$

Since $v \neq 0$ and $\left|\delta_{F}\right|$ is mechanically limited to $22^{\circ}$, the matrix $B_{1}$ is invertible. The objective of this first step is to impose the convergence of the observed state $\hat{X}_{1}$ (derivative of which can be deduced from (6)) to the measured state $\bar{X}_{1}$ thanks to the computation of the vector composed of lateral forces $\left[\begin{array}{ll}\hat{F}_{F} & \hat{F}_{R}\end{array}\right]^{T}$. This can be reached by imposing:

$$
\left[\begin{array}{ll}
\hat{F}_{F} & \hat{F}_{R}
\end{array}\right]^{T}=B_{1}\left(\delta_{F}\right)^{-1}\left(G_{1} \tilde{X}_{1}-A_{1}\left(\hat{X}_{1}\right)\right)
$$

with $\tilde{X}_{1}=\hat{X}_{1}-\bar{X}_{1}$ the observation error and $G_{1}$ a Hurwiz matrix representative of the observer dynamic. Expression (8) indeed imposes the following error dynamics:

$$
\dot{\tilde{X}}_{1}=G_{1} \tilde{X}_{1}
$$

which ensures the exponential convergence of observation error to zero. As a result, since they ensure a satisfactory convergence of model output to the measured one, $\hat{F}_{F}$ and $\hat{F}_{R}$ are representative of the actual forces applied at wheel/ground contact. As a consequence, in view of (2), and considering that cornering stiffnesses are slow varying, a relevant estimation of coefficients $\hat{C}_{i}, i \in\{F, R\}$ should satisfy the following relation:

$$
\hat{F}_{i}=\hat{C}_{i} \bar{\beta}_{i}
$$

The second step then consists in adapting $\hat{C}_{i}$ in order to ensure relation (10). This is obtained using a sensitivitybased gradient search algorithm. An adaptation law for cornering stiffnesses can then be written as:

$$
\dot{\hat{C}}_{i}=-\gamma\left(\hat{F}_{i}-\hat{C}_{i} \bar{\beta}_{i}\right) \frac{\partial\left(\hat{F}_{i}-\hat{C}_{i} \bar{\beta}_{i}\right)}{\partial \hat{C}_{i}}=-\gamma\left(\hat{F}_{i}-\hat{C}_{i} \bar{\beta}_{i}\right) \bar{\beta}_{i}
$$

where $\gamma$ is a strictly positive tunable gain. Adaptation law (11) is then able to adapt on-line the cornering stiffnesses $\hat{C}_{F, R}$, ensuring a relevant adequacy between dynamic model (3) fed with force expression (2) and the preliminary sideslip angle estimation.

\section{Dynamic sideslip angle estimation}

Once the cornering stiffnesses are known, as well as the roll angle is estimated, the observation of sideslip angles using a dynamic model can be proceeded. This is achieved by considering the new state vector $X_{2}=\left[\begin{array}{lll}\dot{\theta}_{2} & \beta_{2} & \sin \alpha\end{array}\right]$ composed of the yaw rate, the global sideslip angle, and the sine of the roll angle. Again, a measurement $\bar{X}_{2}$ of this state is available. Considering dynamic model (3) and the assumption of a small global sideslip angle, the derivative of the state vector can be expressed as:

$$
\dot{X}_{2}=A_{2}\left(C_{F}, C_{R}\right) X_{2}+B_{2}\left(C_{F}, C_{R}\right) \delta_{F}
$$

where:

$$
\begin{gathered}
A_{2}=\left[\begin{array}{ccc}
\frac{-L_{F}^{2} C_{F}-L_{R}^{2} C_{R}}{v I_{z}} & \frac{-L_{F} C_{F}+L_{R} C_{R}}{I_{z}} & 0 \\
-\frac{L_{F} C_{F}-L_{R} C_{R}}{v^{2} m}-1 & -\frac{C_{F}+C_{R}}{v m} & \frac{g}{v} \\
0 & 0 & 0
\end{array}\right], \\
B_{2}=\left[\begin{array}{c}
\frac{L_{F} C_{F}}{I_{z}} \\
\frac{C_{F}}{v m} \\
0
\end{array}\right]
\end{gathered}
$$

As $\alpha$ has been previously estimated, the last line of matrices $A_{2}$ and $B_{2}$ is only composed of zeros. An observer equation associated with model (3) can then be defined as:

$$
\dot{\hat{X}}_{2}=A_{2}\left(\hat{C}_{F}, \hat{C}_{R}\right) \hat{X}_{2}+B_{2}\left(\hat{C}_{F}, \hat{C}_{R}\right) \delta_{F}+G_{2} \tilde{X}_{2}
$$


where $\tilde{X}_{2}=\hat{X}_{2}-\bar{X}_{2}$ is the observation error and $G_{2}$ is a gain matrix defining the observer dynamics.

Provided that $\left(\hat{C}_{F}, \hat{C}_{R}\right)$ are close to $\left(C_{F}, C_{R}\right)$, it can be deduced from (14) and (12) that this observer leads to the differential equation (15):

$$
\dot{\tilde{X}}_{2}=\left(A_{2}+G_{2}\right) \tilde{X}_{2}
$$

The $G_{2}$ matrix defined by:

$$
G_{2}=\left[\begin{array}{ccc}
g_{1,1} & g_{1,2} & 0 \\
g_{2,1} & g_{2,2} & 0 \\
0 & 0 & 0
\end{array}\right]
$$

is then chosen so that the matrix $\left(A_{2}+G_{2}\right)$ ensures the exponential convergence of the two first components of $\tilde{X}_{2}$ to zero. The choice for $G_{2}$ permits to favor the convergence of the first term $\dot{\hat{\theta}}_{2}$ related to the measured yaw rate, rather than the other one related to the estimated global sideslip angle (which is slowly reactive). As a result, a new observation $\hat{\beta}_{2}$ of the global sideslip angle is then available. Finally, using this last estimation, both front and rear sideslip angles can be deduced using the two last equations of dynamic model (3):

$$
\left\{\begin{array}{l}
\beta_{R}^{D y n}=\arctan \left(\tan \hat{\beta}_{2}-\frac{L_{R} \dot{\hat{\theta}}_{2}}{v \cos \left(\hat{\beta}_{2}\right)}\right) \\
\beta_{F}^{D y n}=\arctan \left(\tan \hat{\beta}_{2}+\frac{L_{F} \dot{\hat{\theta}}_{2}}{v \cos \left(\hat{\beta}_{2}\right)}\right)-\delta_{F}
\end{array}\right.
$$

This new expression for sideslip angle estimation is obtained thanks to dynamic representations. This ensures a reactive sideslip angle estimation, improving the tracking capabilities with respect the case where the control law was fed by preliminary sideslip angle observer.

\section{CONTROL LAW DESCRIPTION}

The control law associated with extended kinematic model (1) is deeply detailed in [9], and only briefly described in this section. It is based on an exact linearization of the proposed kinematic model, specifically a conversion into a chained form (see [2]). The control expression for steering angle $\delta_{F}$ is then decomposed into two parts. The first is reactive and relies mainly on current errors and observed sideslip angles. It can then be considered as adaptive. The second term consists in a predictive curvature servoing using the knowledge of the reference trajectory. Based on Model Predictive Control theory (see [16]), it considers the future path curvature in order to anticipate for low level actuator delays and mobile robot inertia. As a result, an expression for this control is:

$$
\delta_{F}=\delta_{\text {Traj }}^{\text {Pred }}+\delta_{\text {Deviation }}\left(y, \tilde{\theta}, \beta_{F}, \beta_{R}\right)
$$

The reactive (and adaptive) term $\delta_{\text {Deviation }}\left(y, \tilde{\theta}, \beta_{F}, \beta_{R}\right)$ relies on the observed sideslip angles and can be fed by different observers. In the result section, several configurations are used to investigate the interest of the proposed observer:

- Case A - Without sliding accounted. In order to highlight the importance of taking sliding into account, this control law considers that sideslip angles are negligible: $\left(\beta_{F}, \beta_{R}\right)=(0,0)$

- Case B - Extended kinematic observer. In this configuration, the preliminary observation is only considered: sideslip angles estimated via the kinematic based observer are input in the control law: $\left(\beta_{F}, \beta_{R}\right)=\left(\bar{\beta}_{F}, \bar{\beta}_{R}\right)$

- Case C - Dynamic observer without slope accounted. In order to investigate the effect of the terrain geometry, the control law is here fed with dynamic sideslip angle observation $\left(\beta_{F}, \beta_{R}\right)=\left(\beta_{F}^{D y n}, \beta_{R}^{D y n}\right)$, but without considering the mobile robot inclination: $\alpha=0$

- Case D - Dynamic observer with slope accounted. This consists in using the control law with the observation strategy proposed in this paper: $\left(\beta_{F}, \beta_{R}\right)=$ $\left(\beta_{F}^{D y n}, \beta_{R}^{D y n}\right)$.

\section{EXPERIMENTAL RESULTS}

\section{A. Experimental robot and on-boarded sensors}

In order to study the capabilities of the proposed adaptive control strategy on uneven ground, the mobile robot depicted in figure 5 is used. This electrical vehicle can reach an $8 \mathrm{~m} / \mathrm{s}$ velocity and is able to climb slopes up to $30^{\circ}$. Its weight is $450 \mathrm{Kg}$ (comprising on-boarded sensors).

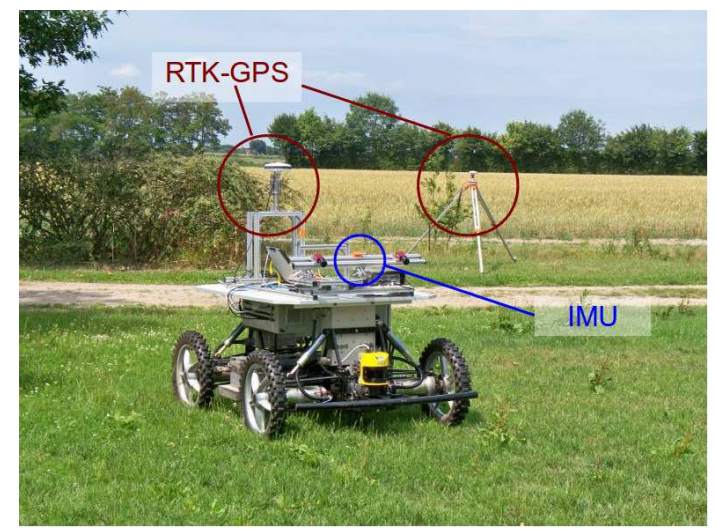

Fig. 5. Experimental robot and embedded sensors

The sensors used in the framework of this paper are:

- An RTK-GPS (Magellan Proflex 500 receiver). The mobile antenna is settled up to the middle of the rear axle, providing an absolute position accurate to within $\pm 2 \mathrm{~cm}$ with a $10 \mathrm{~Hz}$ sampling frequency. Thanks to this sensor, deviations with respect to the desired path $y$ and $\tilde{\theta}$, as well as the velocity $v$ are known. They are computed thanks to the absolute position of the robot with respect to the available reference trajectory composed of successive coordinates (previously computed or recorded during a preliminary manual driving). The control law fed with the preliminary estimation of sideslip angles can then be applied, and robot path tracking at low speed can be achieved. It is assumed that there is no multi-path interferences depreciating the position accuracy delivered by the GPS. This problem could be taken into account by using punctually inertial 
navigation, but such a situation is not addressed in this paper.

- A low cost IMU (Xsens MTi device). This sensor provides three accelerations and three angular velocities, allowing to estimate lateral inclination and feed the observer with the yaw rate $\dot{\theta}$. This sensor can be used solely to obtain a direct estimation of the robot attitude (3D orientation) up to some drift. Some devices permit to combine IMU data with GPS ones, via a proprietary algorithm. In this paper, IMU raw data are favored, so that this sensor can be used with any considered robot. Other sensors depicted in figure 5 (cameras and laser) are not used in the application described in this paper. With this testbed, two sets of experiments are proposed. The first one is achieved at low speed on a slope, in order to compare the different approaches. The second is performed at high speed $(6 \mathrm{~m} / \mathrm{s})$ on a terrain with a varying geometry and with a curved-shape for the path to be followed. This second set permits to show the efficiency of the proposed algorithm.

\section{B. Comparison of observation strategies (low speed)}

This first set of tests consists in tracking a straight line on a varying-sloping field (wet grass). The path to be followed is perpendicular to the slope, inducing a lateral inclination for the mobile robot. As depicted at the top in figure 6, the path to be tracked starts on an almost even ground. Next, the roll angle is rising up to $15^{\circ}$, and then is decreasing. Four path tracking experiments with control law (18) have been achieved with a desired $2 \mathrm{~m} / \mathrm{s}$ velocity, using the four configurations (A to D) described in section IV. Performances are compared at the bottom in figure 6 .

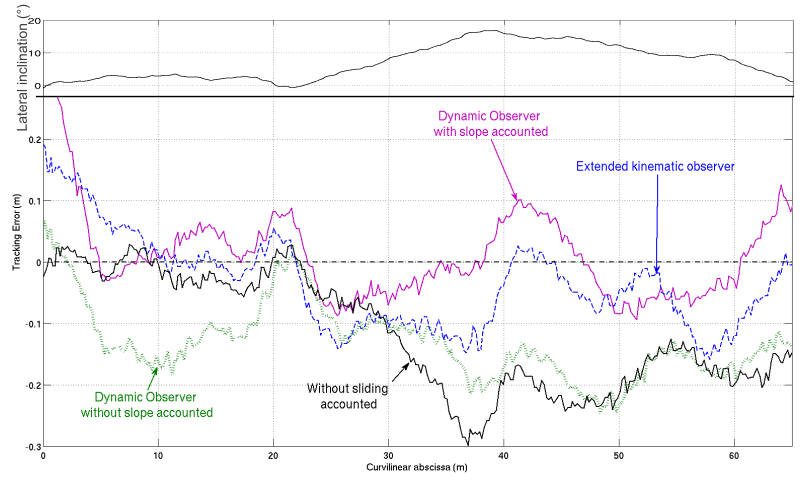

Fig. 6. Tracking error comparison w.r. to observation strategies

It can first be noticed that when a classical control (Case $\mathrm{A}$, depicted in black plain line) is used, the tracking error reaches $-30 \mathrm{~cm}$. This maximal deviation corresponds to the maximum lateral inclination along the reference trajectory. Since the nature of the soil, together with the robot tires, do not ensure perfect grip conditions, the lateral slope generates sliding. Since sideslip angles are neglected, the control law is not able to compensate for such a perturbation and important errors can be observed in relationship with the robot inclination.
On the contrary, the control law with kinematic estimation of sideslip angles (Case B, depicted in blue dotted line) permits to preserve a satisfactory level of accuracy at such a speed $(2 \mathrm{~m} / \mathrm{s})$, as the tracking error stays close to zero. At such a limited speed, the estimated variables $\beta_{F}$ and $\beta_{R}$ ensure ensure that model (1) is consistent with the measured outputs. As a result, the control law is able to compensate for the encountered sliding. Nevertheless, since dynamical effects are neglected, an important deviation (around $-15 \mathrm{~cm}$ ) is recorded between curvilinear abscissas $25 \mathrm{~m}$ and $35 \mathrm{~m}$. This shows the slow reactivity of this approach, compared to the control law based on dynamic observation of sideslip angles and accounting for mobile robot inclination $\alpha$ (Case D reported in magenta plain line in figure 6). The tracking error obtained with the proposed approach indeed converges quickly to zero as soon as the roll angle is observed. A maximal error of $10 \mathrm{~cm}$ is then reached but for a very short moment. This demonstrates the improvement brought by the algorithm proposed in this paper by accounting slope and using a multi-model based observer.

Such performances are explained by the faster observation of sideslip angles, compared to the sole kinematic observer based approach. A comparison of the estimated front sideslip angles $\left(\beta_{F}\right)$ is reported in figure 7 . The estimation obtained using the sole kinematic observer (Case B) is reported in dashed blue line, while the sideslip angle computed from the multi-model observer (Case D) is depicted in magenta plain line. The two estimates are slightly different, highlighting the better reactivity of the algorithm proposed in this paper. It can indeed be noticed that between curvilinear abscissas 25 and $35 \mathrm{~m}$, the value provided by the dynamic observer reaches faster the almost constant $1.5^{\circ}$ value, while the extended kinematic observer supplies delayed values, generating the tracking error pointed out previously. The tracking error in Case B indeed reaches null value (perfect tracking) only when the kinematically estimated front sideslip angle reaches the one dynamically estimated (around $40 \mathrm{~m}, 55 \mathrm{~m}$ and $65 \mathrm{~m}$ ).

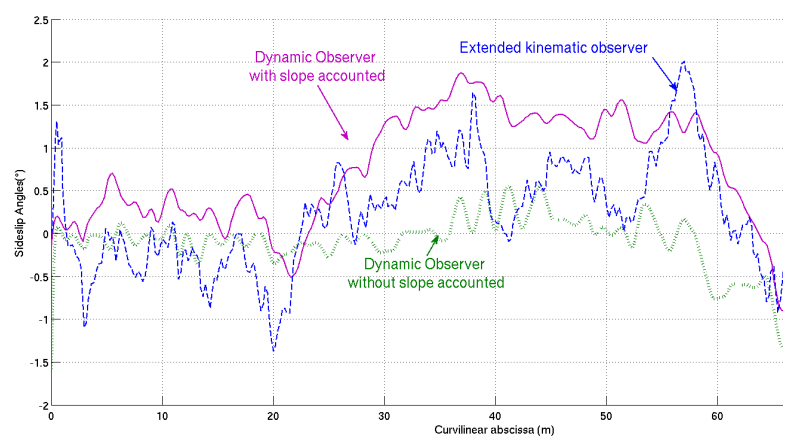

Fig. 7. Comparison of observed front sideslip angles observed

The interest of taking the robot inclination into account in the dynamic observer is also highlighted in both figures 6 and 7 . When satisfactory results were obtained on flat ground (see [10]), the dynamic observation without accounting for the terrain geometry is clearly not suitable when moving on an uneven ground. The tracking error recorded when 
controlling the robot with dynamic estimation of sideslip angles, but neglecting for the lateral inclination $\alpha=0$ (Case $\mathrm{C}$ - results reported in green dotted line in both figures 6 and 7), is similar to the one obtained without sliding accounted (as reported in the figure 6). Since $\alpha$ has been set equal to 0 , the dynamic model (3) cannot generate non-null sideslip angles when the vehicle moves on the sloping part of the trajectory, even if a global sideslip angle $\bar{\beta}$ has been estimated by the preliminary observer (see section III-A). As a result, the dynamic model cannot fit with the actual behavior and the observer is unable to estimate relevant sideslip angles. As it can be seen in figure 7, the front sideslip angle observed in Case $\mathrm{C}$ (when neglecting robot inclination) stays around zero during all the experiment, although the important slope generates non-null actual sideslip angles.

The improvement at the limited speed $2 \mathrm{~m} / \mathrm{s}$ is noticeable, but since sideslip angles do not change quickly, the tracking performances obtained when relying on the kinematic observer might still be regarded as acceptable. At higher speed, sideslip angles are fast varying, and the delay in their estimation if a kinematic observer is used may then lead to control instability. In that case, only the adaptive control law based on the multi-model dynamic observer can ensure meaningful results, as demonstrated in the following section.

\section{Results at high speed on slope}

The second set of experiments consists in tracking the trajectory reported in black plain line in figure 8 at $6 \mathrm{~m} / \mathrm{s}$ on a grass ground. It is composed of a straight line on a terrain with a $20^{\circ}$ slope angle, followed by a harsh bend, achieved on slope and then on even terrain. This path is ended by a small straight line part.

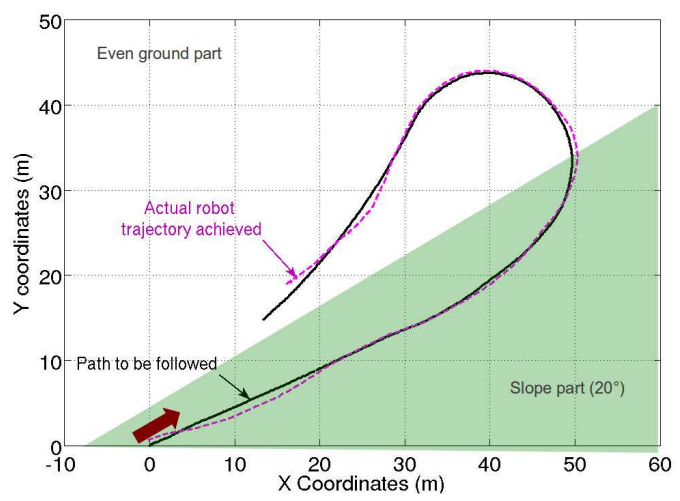

Fig. 8. Reference path and actual trajectory achieved

This trajectory has been followed using the proposed dynamic observer accounting for the robot lateral inclination at $6 \mathrm{~m} / \mathrm{s}{ }^{1}$. The actual trajectory during autonomous path following is reported in magenta line in figure 8 , while the tracking error is plotted in figure 9.

It can be seen that after initialization (up to curvilinear abscissa 20m), the robot is able to reach the reference

\footnotetext{
${ }^{1}$ See the video of the automatic tracking on ftp://ftp.clermont.cemagref.fr/pub/Tscf/Lenain/VideoIros2012/
}

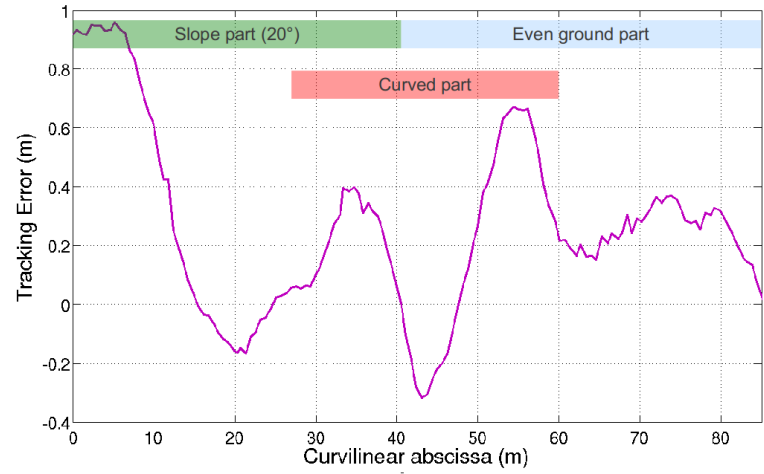

Fig. 9. Tracking error at high speed on slope/even ground at $6 \mathrm{~m} / \mathrm{s}$

trajectory (lateral deviation tends to zero) in the slope part, despite bad grip conditions and harsh dynamical effects (as it can be noticed in the joint video). The inertial effects when entering into the curve, together with the perturbation due to the transition between slope and even parts, generate a transient overshoot at curvilinear abscissa $50 \mathrm{~m}$. This transient lateral deviation is nevertheless quite limited since the maximal tracking error recorded after initialization (the mobile robot does not start on the reference path) is $60 \mathrm{~cm}$ (corresponding at $6 \mathrm{~m} / \mathrm{s}$ to the distance achieved during $0.1 \mathrm{~s})$. This satisfactory result has been obtained thanks to the reactivity of the proposed observer and the consideration of the robot inclination due to the slope. In contrast, the sideslip angle obtained with the kinematic observer present large delays, generating robot oscillations with important errors beyond $2 \mathrm{~m}$. For security reasons, the robot is stopped when the lateral error exceeds $\pm 2 \mathrm{~m}$, so that experimental results obtained in Case B cannot be reported. The bad performances obtained with other configurations (Case A and C), also lead to large errors and then only the results related to Case D (proposed algorithm) is here presented.

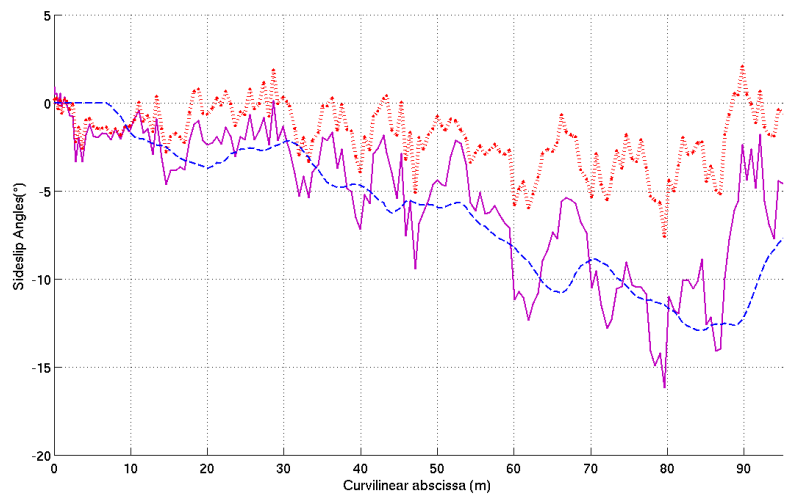

Fig. 10. Comparison of front sideslip angle estimation (Kinematic vs Dynamic estimation)

Nevertheless, the sideslip angles supplied by the kinematic observer can be recorded and are compared in figure 10 to the ones obtained with the dynamic observer (only front sideslip angles are shown). It can be seen in this figure that dynamic estimation (depicted in plain magenta line) is much 
more reactive than kinematic estimation (depicted in blue dashed line). As a result, the control law based only on kinematic estimation (Case B) is unable to compensate for fast sideslip angle variations occurring at high speed. Due to delayed estimation, an oscillating behavior with large errors is recorded. Such a control law is then not effective in these conditions, since dynamic effects are here very important for this robot at the considered speed on such a terrain. The use of constant cornering stiffnesses in the proposed algorithm has also been investigated: it still permits to catch the fast variations in contact conditions, but misestimates the value of sideslip angles. In figure 10, the front sideslip angles estimated by the dynamic observer without updating the cornering stiffnesses (equal to their initial value of $20000 \mathrm{~N} / \mathrm{rad}$ ) is reported in red dotted line. The estimated angle is around the half of the one obtained with on-line adapted cornering stiffnesses. Such an error (more than 5 degrees) leads to a lateral deviation of more than $2 \mathrm{~m}$ at $6 \mathrm{~m} / \mathrm{s}$. Moreover, constant cornering stiffnesses do not permit to account for grip condition variations, which are quite significant in the context addressed in this paper. Finally, only the control law fed with the observer proposed in this paper is relevant to follow the proposed reference trajectory at high speed in the encountered experimental conditions, establishing the capabilities of the approach here detailed.

\section{CONCLUSIONS}

This paper proposes a predictive and adaptive approach for path tracking algorithms, enabling an accurate motion control at high speed in off-road context. It takes advantage of several levels of modeling (extended kinematic and dynamic). The extended kinematic representation is used to derive a control law, efficient as soon as sideslip angles are available. These variables cannot be measured and are consequently observed thanks to an observer taking advantage of kinematic and dynamic models. In addition, the robot lateral inclination is on-line estimated in order to feed the dynamic model. This permits a fast and accurate reconstruction of sideslip angles, ensuring an accurate path tracking, whatever the grip conditions, the terrain geometry and the path to be followed.

This algorithm is dedicated to motion control at high speed (results obtained at $6 \mathrm{~m} / \mathrm{s}$ show the relevancy of the approach). However, moving at such speeds on natural environments may lead to hazardous situations (such as rollover). In the last presented experiment, one of the robot's wheel indeed lifts off during transition between slope and even parts. The proposed control strategy does not ensure the robot integrity with respect to such situations. Future work is then focused on the extension of motion control moderation in order to ensure robot stability. Beyond reactivity, obstacle avoidance is also under investigation, but it requires the knowledge of terrain geometry at high speed, which constitutes a challenging problem. Moreover, the computation of a 3D model implies an accurate pose estimation ( 3 positions and 3 orientations). The use of complete embedded solutions for robot state measurement is then foreseen.

\section{ACKNOWLEDGMENT}

This work is supported by French National Research Agency (ANR), under the grant ANR-07-ROBO-0008 attributed to the FAST project.

\section{REFERENCES}

[1] M. Buehler, K. Iagnemma, and S. Sanjiv, "The 2005 DARPA Grand Challenge: the great robot race," Springer Tracts in Advanced Robotics, vol. 36, 2007.

[2] C. Samson, "Control of chained systems. application to path following and time-varying point stabilization of mobile robots," IEEE Transactions on Automatic Control, vol. 40(1), pp. 64-77, 1995.

[3] C. B. Low and D. Wang, "Robust path following of car-like WMR in the presence of skidding effects," in IEEE international conference on mechatronics, 2005, pp. 864-89.

[4] L. Corradini, T. Leo, and G. Orlando, "Experimental testing of a discrete-time sliding mode controller for trajectory tracking of a wheeled mobile robot in the presence of skidding effects," Journal of Robotic System, vol. 19, no. 4, pp. 177-188, 2002.

[5] M. Ellouze and B. d'Andréa-Novel, "Control of unicycle-type robots in the presence of sliding effects with only absolute longitudinal and yaw velocities measurement." European Journal of Control, vol. 6 , no. 6, pp. 567-584, 2000.

[6] J.-C. Ryu, S. Agrawal, and J. Franch, "Motion planning and control of a tractor with a steerable trailer using differential flatness," ASME Transactions, Journal of Computational and Nonlinear Dynamics, vol. 3, no. 3, pp. $031003.1-8,2008$.

[7] R. Lenain, B. Thuilot, C. Cariou, and P. Martinet, "Rejection of sliding effects in car like robot control: application to farm vehicle guidance using a single rtk gps sensor," in Ieee/rsj international conference on intelligent robots and systems, vol. 4, Las Vegas, Nevada (USA), 2003 pp. 3811-3816.

[8] D. Wang and C. Low, "An analysis of wheeled mobile robots in the presence of skidding and slipping: Control design perspective," in IEEE International Conference on Robotics and Automation (ICRA), Roma, Italy, 2007, pp. 2379-2384.

[9] R. Lenain, B. Thuilot, C. Cariou, and P. Martinet, "High accuracy path tracking for vehicles in presence of sliding. application to farm vehicle automatic guidance for agricultural tasks," Autonomous robots, vol. 21(1), pp. 79-97, 2006.

[10] R. Lenain, B. Thuilot, O. Hach, and P. Martinet, "High-speed mobile robot control in off-road conditions: a multi-model based adaptive approach," in IEEE International Conference on Robotics and Automation, ICRA'11, 2011, p. 6143:6149.

[11] K. L. Talvala, K. Kritayakirana, and J. C. Gerdes, "Pushing the limits: From lanekeeping to autonomous racing," Annual Reviews in Control, vol. 35 , no. 1 , pp. $137-148,2011$.

[12] G. Campion, G. Bastin, and B. d. Andréa-Novel, "Structural properties and classification of kinematic and dynamic models of wheeled mobile robots," in IEEE international conference on robotics and automation, Atlanta, Georgia (USA), 1993, pp. 462-469.

[13] T. Gillespie, Fundamentals of vehicle dynamics. Warrendale, U.S.A.: SAE International, 1992.

[14] H. B. Pacejka, Tire and vehicle dynamics. Society of Automotive Engineers, 2002.

[15] F. Ben Amar and P. Bidaud, "Dynamic analysis of off-road vehicles," in Intern. symp. on experimental robotics, Standford, U.S.A., 1995.

[16] J. Richalet, "Industrial applications of model based predictive control," Automatica, vol. 29, pp. 1251-1574, 1993. 